\title{
Widely Wavelength-tunable Mid-infrared Fluoride Fiber Lasers
}

\author{
Jun Liu, Man Wu, Bin Huang, Pinghua Tang, Chujun Zhao, Deyuan Shen, Dianyuan Fan, and Sergei K. \\ Turitsyn
}

\begin{abstract}
We demonstrate widely wavelength-tunable continuous-wave (CW) and Q-switched $\mathrm{Er}^{3+}$-doped ZBLAN fluoride fiber lasers operating around $3 \mu \mathrm{m}$ enabled by a volume Bragg grating (VBG). In the $\mathrm{CW}$ operation regime, a total wavelength tuning range of over $160 \mathrm{~nm}$ spanning from $2694 \mathrm{~nm}$ to $2854 \mathrm{~nm}$ has been achieved. For the Q-switched mode of operation, a slightly modified resonator configuration, incorporating a passive Q-switcher, topological insulator $\mathrm{Bi}_{2} \mathrm{Te}_{3}$ nanosheets, can produce stable pulse trains with a pulse width of $880 \mathrm{~ns}$ at a repetition rate of $81 \mathrm{kHz}$, while maintaining a wavelength tuning range of $62 \mathrm{~nm}$ from $2762 \mathrm{~nm}$ to $2824 \mathrm{~nm}$ through adjusting the VBG. In both operation regimes, the output spectral width is measured to be $<0.3 \mathrm{~nm}$ (FWHM) over the whole tuning range. Our work both demonstrates the great wavelength-tuning potential of the $\mathrm{Er}^{3+}$-doped fluoride fiber laser, and also paves a way for the development of a range of high performance mid-infrared laser sources.
\end{abstract}

Index Terms-Mid-infrared, wavelength tunable, Q-switched, topological insulator, fiber lasers.

\section{INTRODUCTION}

M id-infrared (Mid-IR) laser sources in the spectral region of 3-5 $\mu \mathrm{m}$ have attracted a great deal of attention due to their potential for applications in a variety of areas, including plastic and polymer processing, non-invasive medical diagnosis, remote sensing, spectroscopy, IR countermeasures and pumps of other longer mid-IR laser sources [1-3]. For most of these applications, there are requirements of a high output power or energy, as well as a good beam quality and

Manuscript received XX, XX. This work is partially supported by the European Horizon 2020-MSCA-IF (Grant no.701493), the National Natural Science Fund Foundation of China (Grant Nos. 61475102, 61505124 and 61505122, 61605166), and the Science and Technology Planning Project of Guangdong Province of China (Grant No. 2016B050501005).

J. Liu, M. Wu, and D. Fan are with SZU-NUS Collaborative Innovation Center for Optoelectronic Science and Technology, and Key Laboratory of Optoelectronic Devices and Systems of Ministry of Education and Guangdong Province, College of Optoelectronic Engineering, Shenzhen University, Shenzhen 518060, China. (e-mail: liu-jun-1987@live.cn).

B. Huang, P. Tang, and C. Zhao are with Laboratory for Micro-/NanoOptoelectronic Devices of Ministry of Education, IFSA Collaborative Innovation Center, School of Physics and Electronics, Hunan University, Changsha 410082, China. (e-mail: cjzhao@hnu.edu.cn).

D. Shen is with Key Laboratory of Micro and Nano Photonic Structures, Department of Optical Science and Engineering, Fudan University, Shanghai 200433, China.

J. Liu and S. K. Turitsyn are with Aston Institute of Photonic Technologies, School of Engineering and Applied Science, Aston University, Birmingham B4

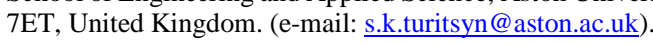

considerable flexibility in operational wavelength, that are difficult to realize simultaneously in conventional bulk solid-state lasers due to the high thermal loading and narrow gain spectrum. In contrast to solid-state ion-doped crystal and other bulk laser oscillators, fiber-based laser sources can provide a promising route to achieving mid-IR laser emissions with superb performance thanks to their compact geometry having a high degree of immunity from thermal effects, distinct power scaling capability without decreasing efficiency or degrading beam quality, and the broad emission spectrum offering a flexibility in the operational wavelength. However, up to now a large gap still exists between the development of fiber lasers operating in the mid-IR wavelength region and those in the near-IR band, mostly due to the lack of efficient mid-IR optical components. Fortunately, mid-IR gain fibers that constitute the main element in a mid-IR fiber laser have been developed in recent years. Typical mid-IR gain fibers, such as rare-earth-ion doped fluoride and chalcogenide-based fibers with good mid-IR transparency and high lasing efficiency, can be fabricated [4-5] and utilized in the high-power fiber lasers at around $\sim 3 \mu \mathrm{m}$ [6-12].

Spectral tunability and narrowing are important features of a laser source, highly desirable in a vast range of applications in science, medicine, spectroscopy, measurements and other fields. The core element in the implementation of high-power narrow-linewidth and tunable laser emissions for various practical applications is an efficient wavelength-selection component. Fiber Bragg gratings (FBGs), characterized by their narrow linewidth and high reflectivity, and above all, convenience of alignment-free operation by avoiding the use of bulky components, are widely used in fiber laser sources to select and tune the lasing wavelength. High-power erbium-doped all-fiber lasers emitting at $2.94 \mu \mathrm{m}$ and $3.44 \mu \mathrm{m}$ have been realized by V. Fortin et al. using FBGs with the corresponding output spectral linewidth of $0.15 \mathrm{~nm}$ and $<0.6$ $\mathrm{nm}$, respectively [6-7]. However, FBGs are not very effective for wavelength selection and spectral narrowing in large mode area fibers, which can support higher-order transverse modes [13]. Besides, FBG tuning based on either thermal effects or mechanical stress is challenging for a wide wavelength range. Another common approach is to use an external feedback cavity incorporating a replica diffraction grating. S. Tokita et al. demonstrated a broadly wavelength-tunable operation of a

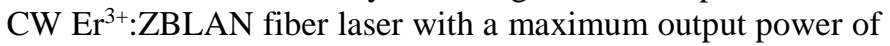
$>10 \mathrm{~W}$ using a diffraction grating with 600 lines $/ \mathrm{mm}$ in the Littrow configuration [9]. The spectral linewidth across the tuning range was measured to be $<1 \mathrm{~nm}$. The replica diffraction 
grating can supply a wide tuning range limited only by the emission spectrum of the gain medium. However, to narrow the output spectral width below $0.5 \mathrm{~nm}$, the laser setup can be quite large and cumbersome with a relatively large collimated beam size in the external cavity (spectral linewidth $\Delta \lambda \propto$ $\mathrm{dM}^{2} \cos \theta / \pi \omega$, where $d$ is the grating pitch, $\mathrm{M}^{2}$ is the beam quality factor, $\theta$ is the incident angle onto the grating, and $\omega$ is the collimated beam diameter on the grating.) [10-11].

An alternative strategy for wavelength selection and spectral narrowing is to employ a volume Bragg grating (VBG), another type of robust optical element recorded in photo-thermo-refractive (PTR) glass. VBGs combine the advantages of high diffraction efficiency, narrow spectral width, low insertion losses, a high damage threshold, and good thermal stability, which make them very attractive as excellent spectral narrowing and wavelength-selection components in high-power, narrow-linewidth, and wavelength-tunable laser sources. Efficient applications of VBGs for stabilizing and narrowing the laser diodes [14], optical parametric oscillators [15] and solid-state lasers [16] have been frequently demonstrated in past years. More recently, VBGs have also been successfully employed as a wavelength selective element in high-power fiber lasers operating in various spectral regions, such as Yb-doped fiber lasers at $\sim 1 \mu \mathrm{m}$ [17], Er,Yb co-doped fiber lasers at $\sim 1.5-1.6 \mu \mathrm{m}$ [18-19], Raman fiber lasers at $\sim 1.7$ $\mu \mathrm{m}[20]$ and Tm-doped fiber laser at $\sim 2 \mu \mathrm{m}$ [21] with output powers up to $138 \mathrm{~W}, 103 \mathrm{~W}, 11 \mathrm{~W}$ and $112 \mathrm{~W}$, respectively. However, to the best of our knowledge, the wavelength narrowing and selection potential of VBGs in the 2.6-5 $\mu \mathrm{m}$ mid-IR wavelength region has not been reported yet.

In this paper, we demonstrate a broadly tunable mid-IR $\mathrm{Er}^{3+}$-doped ZBLAN fiber laser in CW or Q-switched regimes for the sake of flexibility in mode of operation. Wavelength tuning has been achieved through exploiting an external cavity containing a VBG with a peak reflectivity at $2950 \mathrm{~nm}$. In the $\mathrm{CW}$ regime, we have demonstrated an overall tuning range of $160 \mathrm{~nm}$ from $2694 \mathrm{~nm}$ to $2854 \mathrm{~nm}$. Further power scaling has been performed at the operating wavelength of $2788.8 \mathrm{~nm}$ with the best lasing performance, resulting in a maximum output power of up to $2.1 \mathrm{~W}$ and the corresponding slope efficiency of $22.5 \%$ with respect to the incident pump power. In the Q-switched regime enabled by a passive saturable absorber (SA) based on a topological insulator (TI): $\mathrm{Bi}_{2} \mathrm{Te}_{3}$ nanosheets, stable pulse trains have been obtained with a maximum average output power of $0.459 \mathrm{~W}$ and a corresponding pulse width of $880 \mathrm{~ns}$. The operating wavelength can be tuned from $2762 \mathrm{~nm}$ to $2824 \mathrm{~nm}$, covering a tuning range of $62 \mathrm{~nm}$. The output spectral width is measured to be $<0.3 \mathrm{~nm}$ over the whole tuning range in both operation regimes, limited by the resolution of the monochromator. To the best of our knowledge, this is the first time that VBGs have been applied in the CW or pulsed mid-IR tunable fiber-based laser sources with a wavelength greater than $2.6 \mu \mathrm{m}$, and our results can provide new insight into possible designs of novel mid-infrared tunable fiber laser sources.

\section{EXPERIMENTAL SETUP}

The schematic diagram of the tunable $\mathrm{Er}^{3+}$-doped ZBLAN fiber laser is shown in Fig. 1. The pump light was provided by a fiber-coupled laser diode, with a $105 \mu \mathrm{m}$ core diameter and a numerical aperture (NA) of 0.22 , delivering output power of up to $40 \mathrm{~W}$ at $975 \mathrm{~nm}(<2 \mathrm{~nm}$ FWHM). The gain medium is a double clad $6 \mathrm{~mol} . \% \mathrm{Er}^{3+}$-doped ZBLAN fiber (FiberLabs) with a core diameter of $32 \mu \mathrm{m}$ and a NA of 0.12 , surrounded by a circular-shaped inner-cladding of $\sim 300 \mu \mathrm{m}$ diameter and 0.51 NA. The pump absorption coefficient was measured to be $\sim 4$ $\mathrm{dB} / \mathrm{m}$ in the cladding-pumped ZBLAN fiber through the cut-back method. Therefore, a fiber length of $4.3 \mathrm{~m}$ was selected to guarantee sufficient pump absorption. The pump radiation was collimated and focused into the inner-cladding of the gain fiber through one dichroic mirror (M1) by using two antireflection-coated plano-convex lenses (L1 and L2) of 25 $\mathrm{mm}$ and $50 \mathrm{~mm}$ focal lengths, respectively. The measured pump launch efficiency was approximately $90 \%$. The pump launch end of the gain fiber was perpendicularly cleaved and the opposite side adjacent to the external cavity was angle cleaved at $\sim 8^{\circ}$ to suppress the broadband feedback from the two uncoated fiber facets. Both end sections were carefully mounted in water cooled $\mathrm{V}$-groove heat sinks to prevent possible thermal damage to the fiber coating.

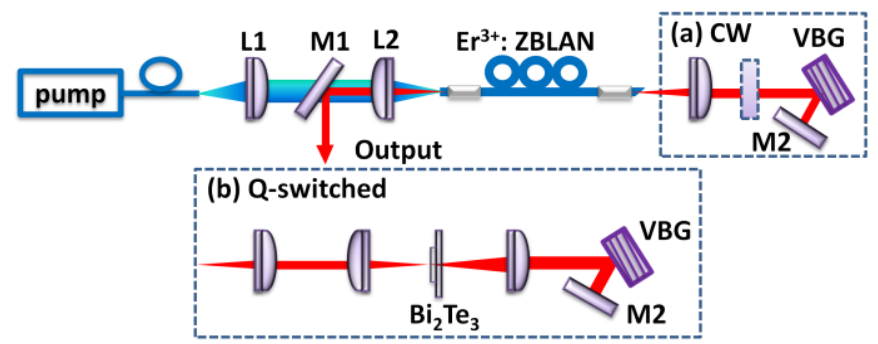

Fig. 1. Schematic diagram of the tunable $\mathrm{Er}^{3+}$ :ZBLAN fiber laser. External feedback cavity incorporating the VBG in the $\mathrm{CW}$ operation regime (a), and in the Q-switched mode of operation (b).

The lasing feedback was provided by the $\sim 4 \%$ Fresnel reflection from the perpendicularly cleaved fiber end (functioned as an output coupler) and a specific external feedback cavity depending on the operation regime (CW or Q-switched) except for the same VBG based wavelength tuning section. The VBG (OptiGrate Corp.) used in the experiment has a center wavelength of $2950 \mathrm{~nm}$ with a peak diffraction efficiency of $>90 \%$ and spectral selectivity of $<4 \mathrm{~nm}$ (FWHM), which was wrapped with a layer of indium foil $(0.1 \mathrm{~mm}$ in thickness) and then mounted in a copper heat sink to ensure good heat removal. In the $\mathrm{CW}$ mode of operation, the external cavity also included an antireflection-coated $\mathrm{CaF}_{2}$ lens with a focal length of $20 \mathrm{~mm}$ that can shape a collimated laser beam of $\sim 5 \mathrm{~mm}$ diameter to be comparable with the $8 \mathrm{~mm} \times 6 \mathrm{~mm}$ clear aperture of the VBG. In the Q-switched operation regime, another two antireflection-coated $\mathrm{CaF}_{2}$ lenses with corresponding focal lengths of $20 \mathrm{~mm}$ and $25 \mathrm{~mm}$ were selected and added to the external cavity to obtain an appropriate focal spot onto the $\mathrm{Bi}_{2} \mathrm{Te}_{3} \mathrm{SA}$, and a comparable collimated laser spot with the VBG simultaneously. A dichroic mirror (M1) was positioned at $45^{\circ}$ in the pump path to extract the laser output. 
In both the $\mathrm{CW}$ and Q-switched operation regimes, wavelength tuning in the $\mathrm{Er}^{3+}$-doped ZBLAN fiber laser was realized by adjusting the angles of both the VBG and the followed HR plane mirror M2 incorporated in the external feedback cavity.

The output spectrum was measured using a calibrated monochromator (Princeton instrument, Acton SP2300) with a resolution of $\sim 0.3 \mathrm{~nm}$ at $\sim 3 \mu \mathrm{m}$ wavelength band. A real-time oscilloscope with a bandwidth of $4 \mathrm{GHz}$ (Agilent Technol., DSO9404A) together with a HgCdTe detector (VIGO System, PCI-9, < 2 ns rise-time at 1-9 $\mu \mathrm{m}$ ) were simultaneously used to monitor the pulse train.

\section{III.RESULTS AND DISCUSSION}

\section{A. CW operation regime}

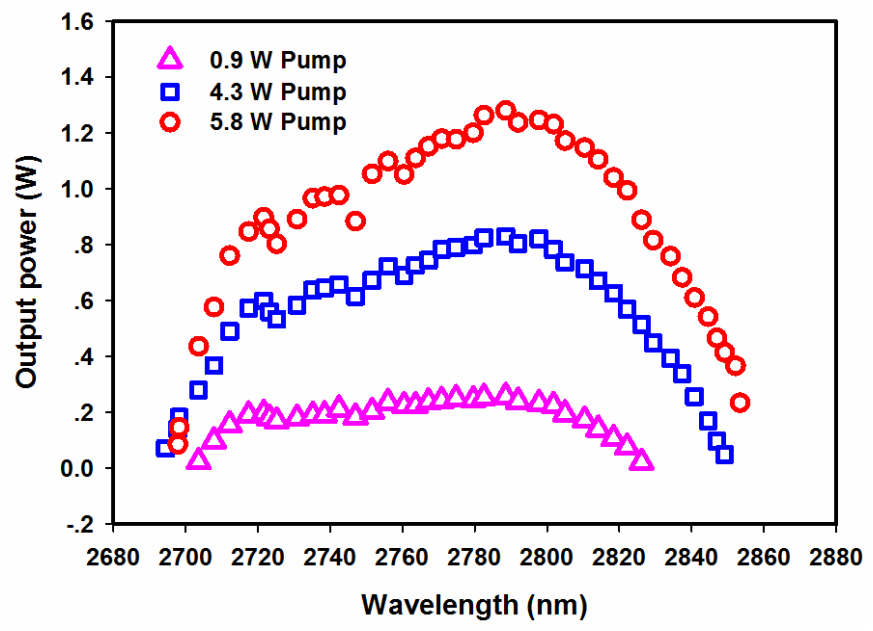

Fig. 2. Output powers as a function of the tuning wavelength for different pump powers.

We first evaluated the lasing characteristics of the tunable $\mathrm{Er}^{3+}$-doped ZBLAN fiber laser in the $\mathrm{CW}$ regime. Figure 2 shows the laser output powers as a function of the tuning wavelength for different pump powers of $0.9 \mathrm{~W}, 4.3 \mathrm{~W}$ and 5.8 $\mathrm{W}$, respectively. It can be seen that the operating wavelengths are all around $2790 \mathrm{~nm}$ for the highest output power under different pump levels, corresponding to the gain peak of the $\mathrm{Er}^{3+}$-doped ZBLAN fiber. Besides, tuning curves at lower pump powers tend to be flatter than that at high pump levels, combined with a sharp drop in output power at the edges of the tuning range for all three curves. For the extremely short wavelength tuning range, lasing became progressively more difficult due to a higher pump threshold resulting from increased reabsorption loss and the reduced reflectivity of the VBG aligned with a large incident angle. The origin of the reduction in power towards the long-wavelength side can be explained by the reduced gain and higher loss at these wavelengths. At the relatively low pump power of $0.9 \mathrm{~W}$, a wavelength tuning range of $124 \mathrm{~nm}$ from $2703 \mathrm{~nm}$ to $2827 \mathrm{~nm}$ was obtained. When the pump power increased to $4.3 \mathrm{~W}$, the tuning range was enlarged to be $156 \mathrm{~nm}$ spanning from 2694 $\mathrm{nm}$ to $2850 \mathrm{~nm}$. A slight redshift of the entire wavelength tuning band occurred at the increased pump power of $5.8 \mathrm{~W}$, covering a tuning range of $157 \mathrm{~nm}$ from 2697 to $2854 \mathrm{~nm}$ with a maximum output power of $1.28 \mathrm{~W}$ at $2788.6 \mathrm{~nm}$. This shift towards the long-wavelength side at high pump levels can be attributed to the increased reabsorption effect for the accumulated large residual populations in the lower laser level. we did not observe any obvious shrinkage of wavelength tuning range with increasing pump power as reported in Ref. [8] and [9] where the high pump intensities applied can further enhance the reabsorption effect. In contrast, in our experiment with relatively moderate pump powers the wavelength tuning range at lower pump powers suffered from much narrower tuning range because lasing at the extremely short wavelength was depressed owing to the weak laser gain and, specifically, much higher insertion loss of the VBG aligned at a large incident angle. In addition, it is worth noting that a wavelength tuning span of as large as $256 \mathrm{~nm}$ for the VBG (from VBG's center wavelength of $2950 \mathrm{~nm}$ to the shortest tuned wavelength of $2694 \mathrm{~nm}$ ) could be realized throughout the tuning process, which demonstrates the VBG's distinct wavelength-tuning capability in tunable laser sources.

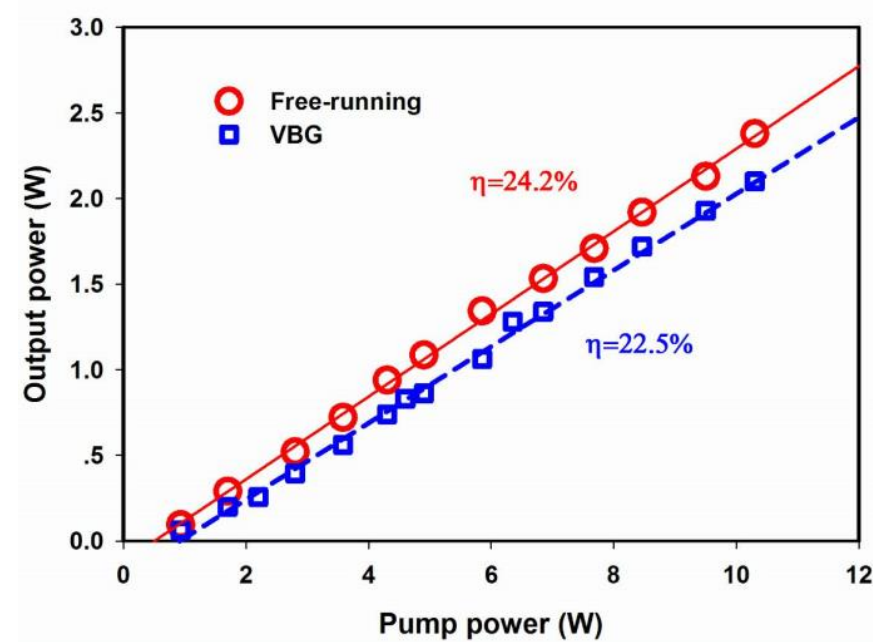

Fig. 3. Output powers as a function of the pump power from the $\mathrm{Er}^{3+}$-doped ZBLAN fiber laser under the free-running and tunable mode of operation.

Output power scaling was only examined at the operating wavelength with the highest efficiency. Figure 3 shows the output power as a function of the pump power when the operating wavelength was locked at $2788.8 \mathrm{~nm}$ using the VBG aligned at a specific incident angle. A maximum output power of $2.1 \mathrm{~W}$ was generated for the incident pump power of $10.3 \mathrm{~W}$, corresponding to a slope efficiency of $22.5 \%$ with respect to the incident pump power. For comparison, we also evaluated the lasing characteristics of the $\mathrm{Er}^{3+}$-doped ZBLAN fiber laser in the free-running operation regime (without the wavelength-selection element). Output power up to $2.4 \mathrm{~W}$ was achieved at the same pump power of $10.3 \mathrm{~W}$ with a slope efficiency of $24.2 \%$ with respect to the incident pump power. The relatively poorer performance of the $\mathrm{Er}^{3+}$-doped ZBLAN fiber laser in the tunable mode in terms of output power and slope efficiency can be attributed to the additional cavity loss induced by the VBG. Nevertheless, the rapid development of the VBG fabrication technology will definitely further increase 
their peak reflectivity and reduce the insertion losses in the mid-IR wavelength region, which can facilitate it bridging the gap between the two operation regimes. Good long-term power stability remained throughout the whole tuning range and the power scaling process with output power fluctuations of less than $1 \%$ over a timescale of 2 hours.
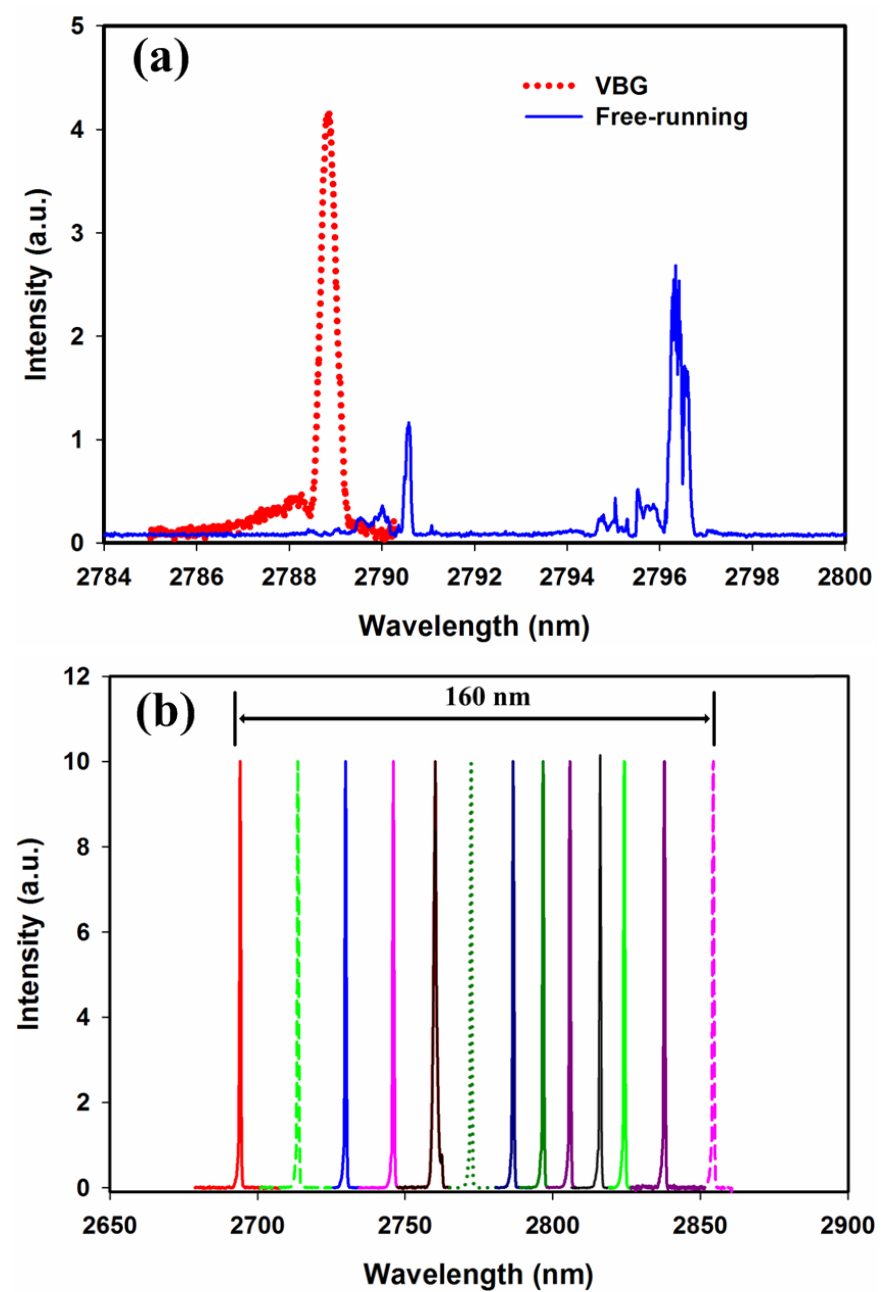

Fig. 4. Laser output spectra of the $\mathrm{Er}^{3+}$-doped ZBLAN fiber laser in typical wavelength-tunable (Red Dot) operation regime and free-running (Blue Line) mode of operation for comparison (a), and measured laser output spectra at different tuning wavelengths in a normalized linear scale (b).

With the main priority being to ensure excellent long-term operational reliability we did not run the $\mathrm{CW}$ tunable fiber laser system at full pump power to test and characterize the high power lasing performance. Actually, prior to the current tunable experiment, several power scaling experiments were performed and we found that once the pump power was increased up to around $20 \mathrm{~W}$, the output power of near $5 \mathrm{~W}$ began to decline sharply after operating for several minutes due to the catastrophic destruction of the fiber tip near the pump launch end. To mitigate the detrimental effect of fiber tip degradation at high-power operation, stable end caps should be used to protect the exposed fiber facets from $\mathrm{OH}$ contamination in the atmosphere that would ultimately result in fiber failure [22]. In our future work, the current laser configuration will be optimized to guarantee the stable operation at high power levels.
The measured laser output spectra in the free-running and wavelength-locked operation regimes are shown in Fig. 4 (a), from which we can see that in the free-running operation regime, the emission spectrum is not very clean and relatively broad spanning from $2789 \mathrm{~nm}$ to $2797 \mathrm{~nm}$ with several obvious spectral peaks. In contrast, the output spectrum in the case of VBG-locked operation exhibits a clear single peak with a similar spectral width of $\sim 0.3 \mathrm{~nm}$, which is comparable to the resolution of the monochromator in this wavelength region. We argue that the actual linewidth of the output spectrum is less than $0.3 \mathrm{~nm}$ and can be accurately measured by a scanned Fabry-Perot interferometer with an appropriate free-spectral range in future. The comparison of the output spectra in the free-running and wavelength-locked operation regimes demonstrate that VBGs perform very well for applications in mid-IR fiber laser sources as wavelength-selection and spectral narrowing elements. Figure 4 (b) shows the measured spectral profiles at different tuned wavelengths in a linear scale. Similarly, the output linewidth of the tunable $\mathrm{Er}^{3+}$-doped ZBLAN fiber laser remained approximately constant over the whole tuning range at $<\sim 0.3 \mathrm{~nm}$, only limited by the resolution of the monochromator.

\section{B. $\quad Q$-switched operation regime}

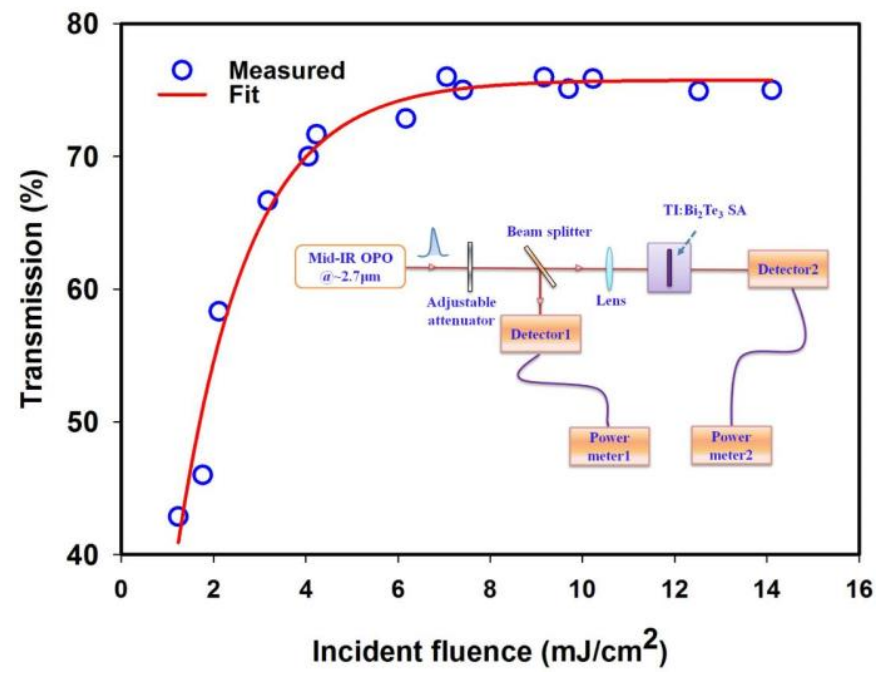

Fig. 5. Measured nonlinear saturable absorption curve of the $\mathrm{Bi}_{2} \mathrm{Te}_{3} \mathrm{SA}$ used in the Q-switched regime; Inset: schematic diagram of the experimental setup for measuring TI's nonlinear saturable absorption.

To validate the wavelength flexibility of the fiber laser in different operation modes, we also constructed a passively Q-switched $\mathrm{Er}^{3+}$-doped ZBLAN fiber laser with a slightly different external feedback cavity, and investigated the Q-switched lasing performance, especially the wavelength tuning characteristics using the same VBG. Passively Q-switched fiber lasers based on SAs have been recognized as an ideal approach to generate high energy pulses owing to their compactness, high efficiency and good beam quality, in contrast to the actively modulated counterpart. A variety of SAs have been investigated and applied in the Q-switched fiber laser sources emitting in the $\sim 3 \mu \mathrm{m}$ wavelength region, such as ion-doped crystals [23], semiconductor saturable absorption mirrors (SESAMs) [24], graphene [25-26], transition metal 
dichalcogenides [27], black phosphorus [28-29] and other graphene-like novel two dimensional (2D) materials. TIs, emerging as one kind of Dirac materials, have attracted considerable attention in recent years as effective ultra-broadband SAs for pulsed laser applications benefiting from their unique broadband saturable absorption property, high damage threshold and tunable modulation depth [30-32].

The Q-switched operation in this experiment was realized based on the TI: $\mathrm{Bi}_{2} \mathrm{Te}_{3} \mathrm{SA}$ included in the external feedback cavity. The $\mathrm{Bi}_{2} \mathrm{Te}_{3}$ nanosheets were synthesized via a solvothermal method, and then dispersed in an ethanol solution. The dispersed solution was then transferred onto an anti-reflection coated $\mathrm{CaF}_{2}$ substrate by drop coating and air drying to prepare the SA. More details about the synthesis of $\mathrm{Bi}_{2} \mathrm{Te}_{3}$ nanosheets and the characterizations of the sample can be found in our previous publication [33].

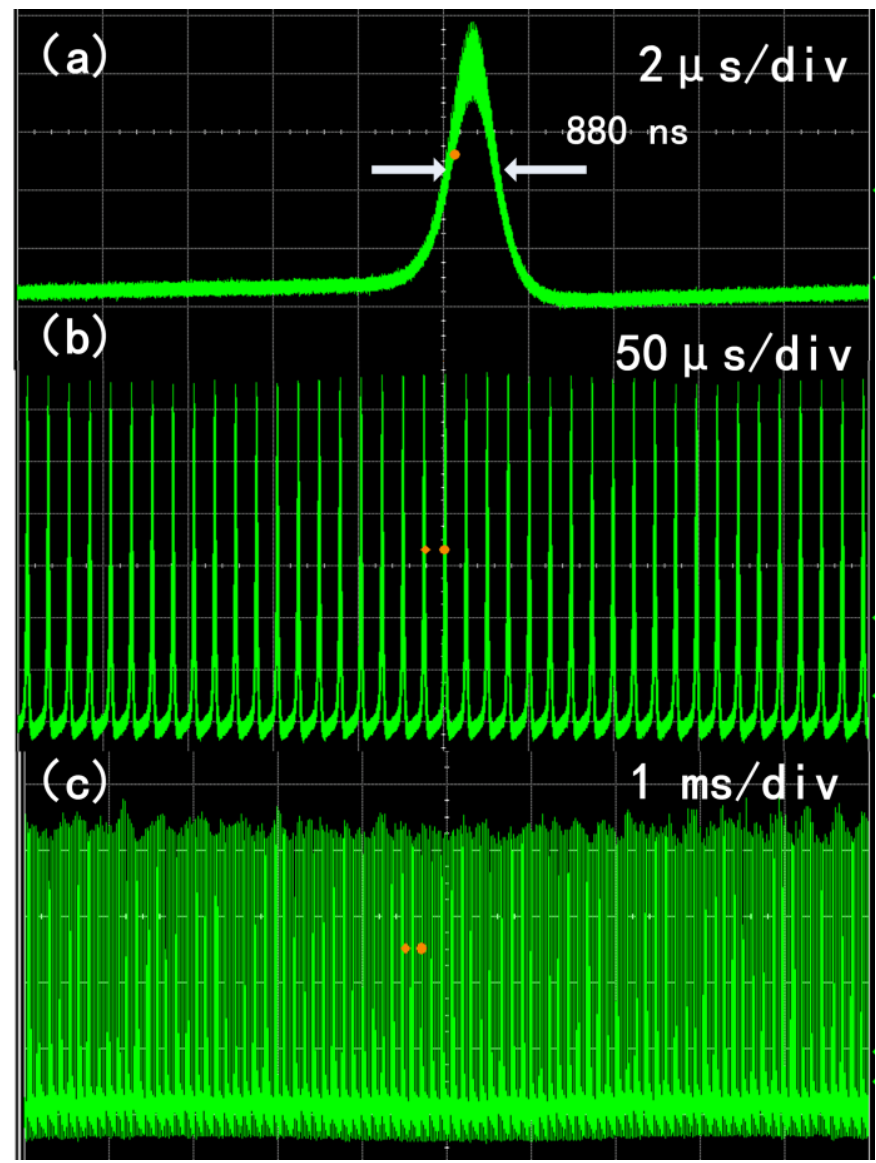

Fig. 6. Temporal profiles of the Q-switched pulse train recorded at different time scales.

The nonlinear saturable absorption of the $\mathrm{Bi}_{2} \mathrm{Te}_{3} \mathrm{SA}$ was measured to confirm the saturable absorption capability near 3 $\mu \mathrm{m}$. The pump source used in the test was an in-house constructed nanosecond pulsed mid-IR OPO system emitting at $\sim 2.7 \mu \mathrm{m}$ [3]. The measured nonlinear absorption curve is shown in Fig. 5, the inset of which depicts the experimental setup. The corresponding saturation pulse influence, modulation depth and non-saturation loss of the TI: $\mathrm{Bi}_{2} \mathrm{Te}_{3} \mathrm{SA}$ were calculated to be $1.54 \mathrm{~mJ} / \mathrm{cm}^{2}, 77.6 \%$, and $24.3 \%$, respectively, according to the measured nonlinear absorption curve.

We then inserted the fabricated $\mathrm{TI}: \mathrm{Bi}_{2} \mathrm{Te}_{3} \mathrm{SA}$ into the external cavity and carefully adjusting its position and the incident angle of the VBG to optimize the pulse stability and average output power. The measured center wavelength of the laser output with the optimal performance in terms of stability and output power locates at $2791.3 \mathrm{~nm}$ with a FWHM spectral linewidth of $<0.3 \mathrm{~nm}$. Figure 6 shows the oscilloscope trace of the Q-switched laser output at the incident pump power of 4.9 $\mathrm{W}$. A maximum average output power of $0.459 \mathrm{~W}$ was obtained with the corresponding pulse width of $880 \mathrm{~ns}$ and a repetition of $81 \mathrm{kHz}$. This is, to the best of our knowledge, the narrowest Q-switched pulses achieved in the mid-IR wavelength region based on TI SAs.

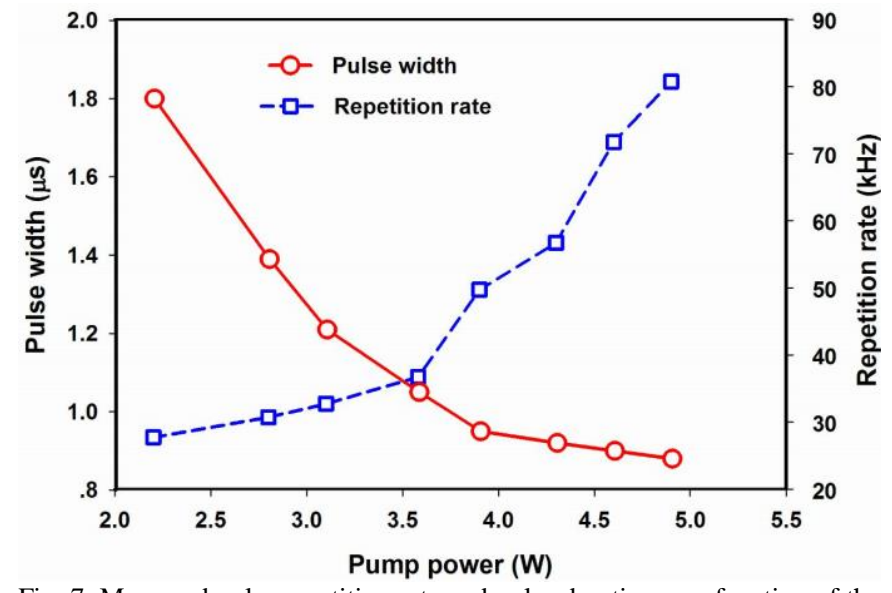

Fig. 7. Measured pulse repetition rate and pulse duration as a function of the incident pump power.

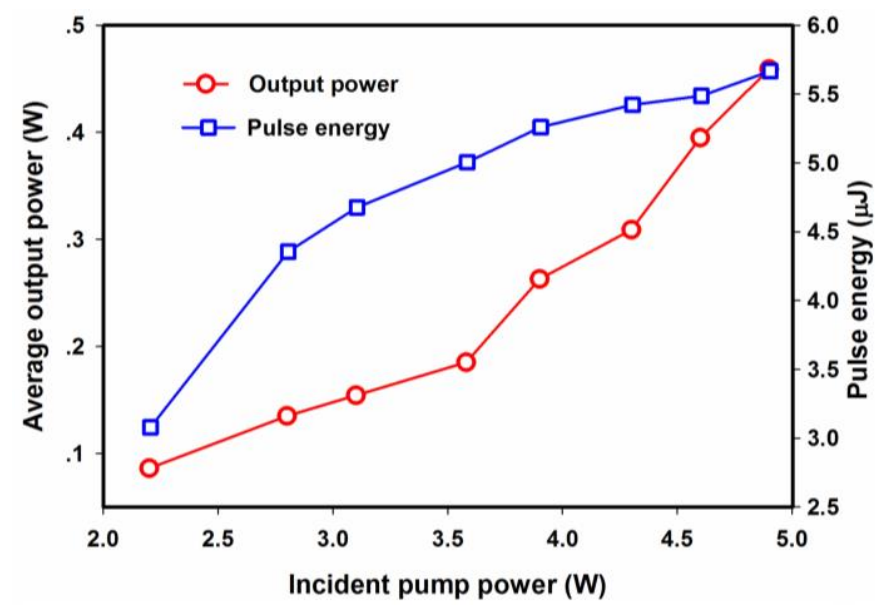

Fig. 8. Output power and corresponding pulse energy as a function of the incident pump power.

The pulse width and repetition rate as a function of the incident pump power are depicted in Fig. 7, from which we can see that the pulse repetition rate increases quasi-linearly with the pump power while the pulse width exhibits a decreasing tendency with respect to the incident pump power. The pulse energy was calculated based on the average output power and pulse repetition rate, as shown in Fig. 8. It can be seen that the average output power increased quasi-linearly with respect to the incident pump power with the corresponding overall slope 
efficiency of $\sim 14 \%$ and a maximum single-pulse energy of 5.7 $\mu \mathrm{J}$ at the pump power of $4.9 \mathrm{~W}$. The pulse-to-pulse amplitude fluctuation was measured to be below $\sim 2 \%$. To further investigate the long-term stability, we also recorded the average output power as well as spectra over a timescale of 2 hours. Neither obvious central wavelength drifting nor power roll-over was observed, which indicates reasonably long-term stability. When we further scaled up the pulse energy by simply increasing the pump power, the Q-switched pulse train became unstable, which is possibly attributed to the thermal load accumulated in the SA.

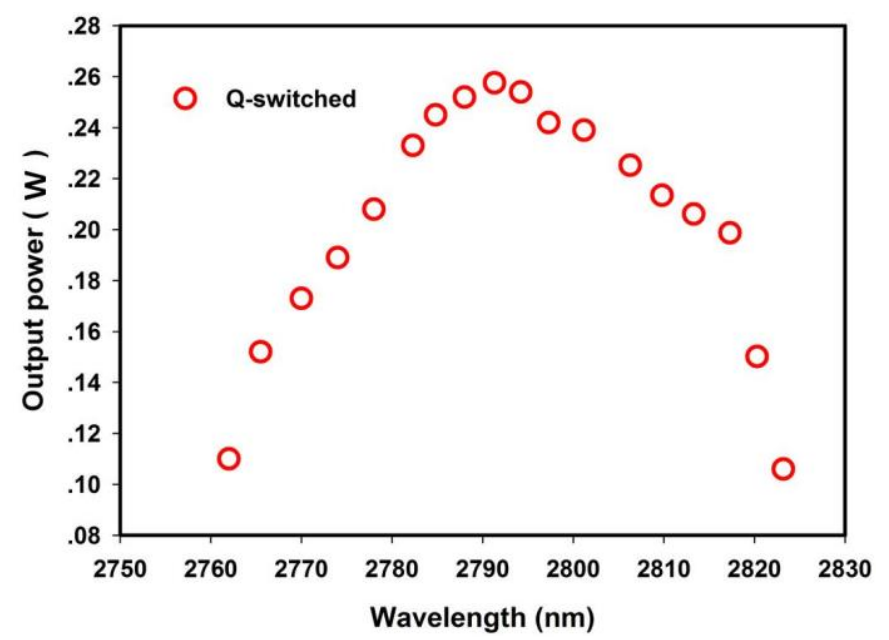

Fig. 9. Output powers as a function of the tuning wavelength at the incident pump power of $3.9 \mathrm{~W}$.

The wavelength tuning characteristics in the Q-switched regime were also evaluated similarly to that in the $\mathrm{CW}$ regime. Continuous wavelength tuning is achieved by adjusting the angles of both the VBG and the following HR plane mirror. To maintain stability of the laser system while performing wavelength tuning in the Q-switched regime, the pump power was kept at $3.9 \mathrm{~W}$. A tuning range of $62 \mathrm{~nm}$ from $2762 \mathrm{~nm}$ to $2824 \mathrm{~nm}$ was obtained with a maximum average output power of $0.26 \mathrm{~W}$ at $2791.3 \mathrm{~nm}$, as shown in Fig. 9. It is worth noting that the tuning range shrank sharply in contrast to that in the $\mathrm{CW}$ regime. Besides, when tuning to both two ends of the curve the pulse train became slightly unstable with a much reduced average output power. This can be attributed to the much higher thermal loading in the gain fiber suffering from intense energy-transfer upconversion and increased heat generation accumulated in the SA in the Q-switched regime. To further extend the wavelength tuning range, the cavity configuration and the SA fabrication technique should be optimized to alleviate these detrimental effects in the future. Similarly, the output spectral width of the tunable Q-switched $\mathrm{Er}^{3+}$ :ZBLAN fiber laser was measured to be $<0.3 \mathrm{~nm}$ over the whole tuning range.

\section{CONCLUSION}

In conclusion, we demonstrated a broadly tunable mid-IR $\mathrm{Er}^{3+}$-doped ZBLAN fiber laser in both $\mathrm{CW}$ and Q-switched regimes through the use of a VBG. Overall tuning ranges of 160 $\mathrm{nm}$ from $2694 \mathrm{~nm}$ to $2854 \mathrm{~nm}$ and $62 \mathrm{~nm}$ from $2762 \mathrm{~nm}$ to 2824 $\mathrm{nm}$ are achieved in the $\mathrm{CW}$ and Q-switched regimes, respectively. For both operation modes, the output spectral width is measured to be $<0.3 \mathrm{~nm}$ over the whole tuning range, limited only by the resolution of the monochromator. Approaches for further extending the wavelength tuning range of the mid-IR fiber laser have been discussed. Further power scaling capability of the tunable $\mathrm{Er}^{3+}$-doped ZBLAN fiber laser will be explored in future. Our experimental results can serve as a convincing demonstration to validate that VBGs can exhibit good performance in mid-IR tunable laser sources as a wavelength-selection component.

\section{REFERENCES}

[1] S. D. Jackson, "Towards high-power mid-infrared emission from a fibre laser," Nat. Photon., vol. 6, no. 7, pp. 423-431, Jun. 2012.

[2] V. Fortin, M. Bernier, N. Caron, D. Faucher, M. E. Amraoui, Y. Messaddeq, R. Vallée, "Towards the development of fiber lasers for the 2 to $4 \mu \mathrm{m}$ spectral region," Opt. Eng., vol. 52, no. 5, pp. 054202, May. 2013.

[3] J. Liu, P. Tang, Y. Chen, C. Zhao, D. Shen, S. Wen, and D. Fan, "Highly efficient tunable mid-infrared optical parametric oscillator pumped by a wavelength locked, Q-switched Er:YAG laser," Opt. Express, vol. 23, no. 16, pp. 20812-20819, Jul. 2015.

[4] H. Heidepriem, T. Foo, R. C. Moore, W. Zhang, Y. Li, T. M. Monro, A. Hemming, and D. G. Lancaster, "Fluoride glass microstructured optical fiber with large mode area and mid-infrared transmission," Opt. Lett., vol. 33, no. 23, pp. 2861-2863, Dec. 2008.

[5] B. Bureau, X. H. Zhang, F. Smektala, J. Adam, J. Troles, H. Ma, C. Boussard-Pldel, J. Lucas, P. Lucas, D. L. Coq, M. R. Riley, J. H. Simmons, "Recent advances in chalcogenide glasses," J. Non-Cryst. Solids, vol. 345-346, pp. 276-283, Oct. 2004.

[6] V. Fortin, M. Bernier, S. T. Bah, and R. Vallée, " $30 \mathrm{~W}$ fluoride glass all-fiber laser at $2.94 \mu \mathrm{m}$,” Opt. Lett., vol. 40, no. 12, pp. 2882-2885, Jun. 2015.

[7] V. Fortin, F. Maes, M. Bernier, S. T. Bah, M. D’Auteuil, and R. Vallée, "Watt-level erbium-doped all-fiber laser at $3.44 \mu \mathrm{m}, "$ Opt. Lett., vol. 41, no. 3, pp. 559-562, Jan. 2016.

[8] X. Zhu and R. Jain, "Compact $2 \mathrm{~W}$ wavelength-tunable Er:ZBLAN mid-infrared fiber laser," Opt. Lett., vol. 32, no. 16, pp. 2381-2383, Aug. 2007.

[9] S. Tokita, M. Hirokane, M. Murakami, S. Shimizu, M. Hashida, and S. Sakabe, "Stable $10 \mathrm{~W}$ Er:ZBLAN fibre laser operating at 2.71-2.88 $\mu \mathrm{m}$," Opt. Lett., vol. 35, no. 23, pp. 3943-3945, Dec. 2010.

[10] D. Hudson, E. Magi, L. Gomes, and S. D. Jackson, "1 W diode-pumped tunable $\mathrm{Ho}^{3+}, \mathrm{Pr}^{3+}$-doped fluoride glass fibre laser," Electron. Lett., vol. 47, no. 17, pp. 985-986, Aug. 2011.

[11] J. Li, D. D. Hudson, and S. D. Jackson, "Tuned Cascade Laser", IEEE Photon. Technol. Lett., vol. 24, no. 14, pp. 1215-1217, Jul. 2012.

[12] P. Tang, Z. Qin, J. Liu, C. Zhao, G. Xie, S. Wen, and L. Qian, "Watt-level passively mode-locked Er3+-doped ZBLAN fiber laser at $2.8 \mu \mathrm{m}$," Opt. Lett., vol. 40, no. 21, pp. 4955-4858, Nov. 2015.

[13] T. Mizunami, T. V. Djambova, T. Niiho, and S. Gupta, "Bragg Gratings in Multimode and Few-Mode Optical Fibers," J. Lightwave Technol., vol. 18, no. 2, pp. 230-235, Feb. 2000.

[14] B. L. Volodin, S. V. Dolgy, E. D. Melnik, E. Downs, J. Shaw, and V. S. Ban, "Wavelength stabilization and spectrum narrowing of high-power multimode laser diodes and arrays by use of volume Bragg gratings," Opt. Lett., vol. 29, no. 16, pp. 1891-1893, Aug. 2004.

[15] J. Saikawa, M. Fujii, H. Ishizuki, and T. Taira, "High-energy, narrow-bandwidth periodically poled $\mathrm{Mg}$-doped $\mathrm{LiNbO}_{3}$ optical parametric oscillator with a volume Bragg grating," Opt. Lett., vol. 32, no. 20, pp. 2996-2998, Oct. 2007.

[16] T. Chung, A. Rapaport, V. Smirnov, L. B. Glebov, M. C. Richardson, and M. Bass, "Solid-state laser spectral narrowing using a volumetric photothermal refractive Bragg grating cavity mirror," Opt. Lett., vol. 31, no. 2, pp. 229-231, Jan. 2006.

[17] P. Jelger, P. Wang, J. K. Sahu, F. Laurell, and W. A. Clarkson, "High-power linearly-polarized operation of a cladding-pumped $\mathrm{Yb}$ fibre laser using a volume Bragg grating for wavelength selection," Opt. Express, vol. 16, no. 13, pp. 9507-9512, Jun. 2008. 
[18] J. Liu, D. Shen, H. Huang, X. Zhang, X. Liu, and D. Fan, "Volume Bragg grating-based tunable $\mathrm{Er}, \mathrm{Yb}$ fiber lasers covering the whole $\mathrm{C}$ - and L-band," IEEE Photon. Technol. Lett., vol. 25 no. 15, pp. 1488-1491, Aug. 2013.

[19] J. W. Kim, P. Jelger, J. K. Sahu, F. Laurell, and W. A. Clarkson, "High-power and wavelength-tunable operation of an $\mathrm{Er}, \mathrm{Yb}$ fiber laser using a volume Bragg grating,” Opt. Lett., vol. 33, no. 11, pp. 1204-1206, Jun. 2008.

[20] J. Liu, D. Shen, H. Huang, C. Zhao, X. Zhang, and D. Fan, "High-power and highly efficient operation of wavelength-tunable Raman fiber lasers based on volume Bragg gratings," Opt. Express, vol. 22, no. 6, pp. 6605-6612, Mar. 2014.

[21]. F. Wang, D. Y. Shen, D. Y. Fan, and Q. S. Lu, "Spectrum narrowing of high power Tm: Fiber laser using a volume Bragg grating," Opt. Express, vol. 18, no. 9, pp. 8937-8941, Apr. 2010.

[22] N. Caron, M. Bernier, D. Faucher, and R. Vallée, "Understanding the fiber tip thermal runaway present in $3 \mu \mathrm{m}$ fluoride glass fiber lasers," Opt. Express, vol. 20, no. 20, pp. 22188-22194, Sep. 2012.

[23] C. Wei, X. S. Zhu, R. A. Norwood, and N. Peyghambarian, "Passively continuous-wave mode-locked $\mathrm{Er}^{3+}$-doped ZBLAN fiber laser at $2.8 \mu \mathrm{m}$," Opt. Lett., vol. 37, no. 18, pp. 3849-3851, Sep. 2012.

[24] A. Haboucha, V. Fortin, M. Bernier, J. Genest, Y. Messaddeq, and R. Vallée, "Fiber Bragg grating stabilization of a passively mode-locked $2.8 \mu \mathrm{m} \mathrm{Er}{ }^{3+}$ : fluoride glass fiber laser," Opt. Lett., vol. 39, no. 11, pp. 3294-3297, Jun. 2014.

[25] C. Wei, X. S. Zhu, F. Wang, Y. Xu, K. Balakrishnan, F. Song, R. A. Norwood, and N. Peyghambarian, "Graphene Q-switched $2.78 \mu \mathrm{m}$ $\mathrm{Er}^{3+}$-doped fluoride fiber laser," Opt. Lett., vol. 38, no. 17, pp. 3233-3236, Sep. 2013.

[26] A. Martinez and Zhipei Sun, "Nanotube and graphene saturable absorbers for fibre lasers," Nat. Photon., vol. 7, no. 11, pp. 842-845, Oct. 2013.

[27] M. Fan, T. Li, S. Zhao, G. Li, H. Ma, X. Gao, C. Kränkel, and G. Huber, "Watt-level passively Q-switched $\mathrm{Er}^{-\mathrm{Lu}_{2} \mathrm{O}_{3}}$ laser at $2.84 \mu \mathrm{m}$ using $\mathrm{MoS}_{2}$," Opt. Lett., vol. 41, no. 3, pp. 540-543, Jan. 2016.

[28] J. Li, H. Luo, B. Zhai, R. Lu, Z. Guo, H. Zhang, and Y. Liu, "Black phosphorus: a two-dimension saturable absorption material for mid-infrared Q-switched and mode-locked fiber lasers," Sci. Rep., vol. 6, pp. 30361, Jul. 2016.

[29] Z. Qin, G. Xie, C. Zhao, S. Wen, P. Yuan, and L. Qian, "Mid-infrared mode-locked pulse generation with multilayer black phosphorus as saturable absorber," Opt. Lett., vol. 41, no. 1, pp. 56-59, Jan. 2016.

[30] C. J. Zhao, H. Zhang, X. Qi, Y. Chen, Z. T. Wang, S. C. Wen, and D. Y. Tang, "Ultra-short pulse generation by a topological insulator based saturable absorber," Appl. Phys. Lett., vol. 101 no. 21, pp. 211106, Nov. 2012.

[31] J. F. Li, H. Y. Luo, L. Wang, C. J. Zhao, H. Zhang, H. P. Li, and Y. Liu, "3- $\mu \mathrm{m}$ mid-infrared pulse generation using topological insulator as the saturable absorber," Opt. Lett., vol. 40, no. 15, pp. 3659-3662, Sep. 2015.

[32] J. S. Ponraj, Z. Xu, S. C. Dhanabalan, H. Mu, Y. Wang, J. Yuan, P. Li,S. Thakur, M. Ashrafi, K. Mccoubrey, Y. Zhang, S. Li, H. Zhang, and Q. Bao, "Photonics and optoelectronics of two dimensional materials beyond graphene," Nanotechnol., vol. 27, no .46, pp. 462001, Oct. 2016.

[33] Q. K. Wang, Y. Chen, L. L. Miao, G. B. Jiang, S. Q. Chen, J. Liu, X. Q. $\mathrm{Fu}, \mathrm{C}$. J. Zhao, and H. Zhang, "Wide spectral and wavelength-tunable dissipative soliton fiber laser with topological insulator nano-sheets self-assembly films sandwiched by PMMA polymer," Opt. Express, vol. 23, no. 6, pp. 7681-2693, Mar. 2015.

Jun Liu received a Ph.D degree in Optical Engineering at the Shanghai Institute of Optics and Fine Mechanics, Chinese Academy of Sciences, in 2014. He is now an assistant professor at SZU-NUS Collaborative Innovation Center for Optoelectronic Science \& Technology at Shenzhen University, and also with Aston Institute of Photonic Technologies, School of Engineering and Applied Science, Aston University, as a Marie-Curie research fellow. His recent research interests and activities include fiber optics, nonlinear optics and laser technology. He has published more than 25 papers in high-standard journals and conferences.

Man Mu is currently working as a Postdoctoral Researcher with SZU-NUS Collaborative Innovation Center for Optoelectronic Science and Technology, College of Optoelectronic Engineering, Shenzhen University, Shenzhen, China. She was awarded a Ph.D. degree at the College of Physics and Microelectronic Science of Hunan University, Changsha, China, in 2014. Her research interests are focused on two-dimensional materials and devices.

Bin Huang is currently pursuing his Ph.D degree at the School of Physics and Electronics of Hunan University. His research interests include fiber lasers, solid-state lasers, and the synthesis of two-dimensional materials.

Pinghua Tang received a B.S. degree in Physics from Hunan Normal University, Changsha, China, in 2010, and his Ph.D degree at the at the School of Physics and Electronics of Hunan University, in 2015. He is now working as a lecturer at Xiangtan University, Xiangtan, China. His research interest is focused on solid-state lasers. He has authored and co-authored over 20 scientific publications in international indexed journals and conferences.

Chujun Zhao received a B. S. degree and a M. S. degree in physics from Hunan University, Changsha, China, in 2002 and 2005, and a Ph. D degree in Optics from the Shanghai Institute of Optics and Fine Mechanics, Chinese Academy of Sciences, Shanghai, China, in 2008. He is currently an Associate Professor with Hunan University, Changsha, China. He has authored and co-authored over 30 journal papers. His current research focuses on ultrafast pulse generation and its applications.

Deyuan Shen received a Ph.D. degree in Physics from Shandong University, Jinan, China, in 1996. He joined the Institute for Laser Science, University of Electro-Communications, Tokyo, Japan, as a Postdoctoral Research Fellow, where he was involved in the field of laser-diode-pumped high-power solid-state laser sources. From 2000 to 2002, he was with the School of Electrical and Electronic Engineering, Nanyang Technological University, Singapore, where he was engaged in developing high-power, visible, and infrared novel laser sources for industrial applications. In 2002, he joined the Optoelectronics Research Centre (ORC), University of Southampton, Southampton U.K., and was promoted to a Senior Research Fellow in 2005. He was engaged in research on eye-safe high-power fiber lasers, fiber-bulk hybrid solid-state lasers, and other novel fiber laser sources. He is currently with the School of Information Science and Engineering, Fudan University, Shanghai, China. His current research interests include power and brightness scaling of cladding-pumped fiber lasers and amplifiers, nonlinear frequency conversion, and laser applications.

Dianyuan Fan received a B.S. degree in Physics from Beijing University, Beijing, China, in 1962, and a M.S. degree in Electronics from the Beijing Institute of Electronics, Chinese Academy of Sciences, Shanghai, China, in 1966. Since 1966, 
he has been with Shanghai Institute of Optics and Fine Mechanics, Chinese Academy of Sciences, Shanghai, China, where he is currently a member of the Chinese Academy of Engineering. He is also with Shenzhen University, Shenzhen, China. He has published over 200 papers in international journals and conferences. His current research interests include laser technology, optical engineering, and nonlinear optics.

Sergei K. Turitsyn graduated from the Department of Physics of the Novosibirsk University, Russia in 1982 and received a Ph.D. degree in Theoretical and Mathematical Physics from the Institute of Nuclear Physics, Novosibirsk, Russia in 1986. From 1992 to 1998, he was with the Institute for Theoretical Physics
I, Heinrich-Heine University, Duesseldorf, Germany, first as a Humboldt Fellow and later as Leader of collaborative projects with Deutsche Telekom. He joined the Photonics Research Group in the School of Engineering and Applied Science, Aston University, United Kingdom in 1998 and is now the Director of the Aston Institute of Photonic Technologies. He received the Royal Society Wolfson Research Merit Award in 2005. In 2011, he received the European Research Council Advanced Grant, in 2014 he received the Lebedev medal by the Rozhdestvensky Optical Society and in 2016 the Aston 50th Anniversary Chair medal. He is a fellow of the Optical Society of America and the Institute of Physics. 